\title{
Epidemiology and treatment of antimicrobial- resistant gram-negative bacteria in Korea
}

\author{
Young Ah Kim ${ }^{1}$ and Yoon Soo Park ${ }^{2}$
}

Departments of ${ }^{1}$ Laboratory Medicine and ${ }^{2}$ Internal Medicine, National Health Insurance Service Ilsan Hospital, Goyang, Korea

\section{Received: February 4, 2018} Accepted: February 12, 2018

\section{Correspondence to}

Yoon Soo Park, M.D.

Department of Internal Medicine, National Health Insurance Service Ilsan Hospital, 100 Ilsan-ro, Ilsandong-gu, Goyang 10444, Korea

Tel: +82-31-900-0979

Fax: +82-31-900-0343

E-mail:yspark@nhimc.or.kr

*This paper was contributed by the Korean Society of Infectious Diseases.

\begin{abstract}
Antimicrobial resistance is becoming one of the greatest challenges to public health worldwide. Infections by antimicrobial-resistant organisms could result in the failure of treatment, increased medical costs, prolonged hospital stays, and an increased socioeconomic burden. Antimicrobial usage in Korea remains heavy, even after much effort to reduce their use. According to the Korean antimicrobial resistance surveillance system, the resistance rates of many bacteria are increasing. The resistance rate of Acinetobacter baumannii to imipenem in Korea increased to $85 \%$ in 2015 , representing a major public threat. The reports of increased carbapenem resistance in Enterobacteriaceae are worrisome. More importantly, some carbapenem-resistant Enterobacteriaceae may result from the production of carbapenemases, which break down carbapenems. There are relatively few treatment options for extensively drug-resistant A. baumannii and carbapenem-resistant Enterobacteriaceae. Most reports are retrospective observational studies. Because there are little published data from randomized controlled trials, more data assessing antimicrobial treatment for extensively drug-resistant A. baumannii and carbapenem-resistant Enterobacteriaceae are needed to make treatment recommendations.
\end{abstract}

Keywords: Drug resistance; Gram-negative bacteria; Epidemiology; Therapeutics

\section{INTRODUCTION}

Antimicrobial resistance (AMR) is becoming one of the greatest challenges to public health worldwide. Infections by antimicrobial-resistant organisms can result in failure of treatment, increased medical costs, prolonged hospital stays, and an increased socioeconomic burden [1]. Antimicrobial usage remains heavy in Korea, even after much effort to reduce their use [2,3]. The AMR burden in Korea is large, especially due to healthcare-associated infections caused by multidrug-resistant organisms (MDROs) [4].

The discovery of antimicrobials was an epochal advance in 2 oth century medicine, but the efficacy of most antimicrobials is decreasing progressively with continuously evolving AMR; this could terminate in a 'post-antibiotics era' in which common infections may be lethal with no available treatment options [5]. In August 2016, the Korean Ministry of Health and Welfare established the Korean National Action Plan on AMR [6]. The action plan has six objectives: (1) to promote the prudent use of antimicrobial medicines; (2) to prevent the spread of AMR; (3) to strengthen the surveillance system; (4) to improve awareness; (5) to strengthen research and development; and (6) to enhance international collaboration. Prudent antimicrobial prescriptions, based on evidence and the current epidemiology of AMR, is the key to solving the AMR problem, which is an urgent public health concern. 
We reviewed the current epidemiology of MDROs in Korea, focusing on Gram-negative bacilli (GNB), because the recent sharp rise in the isolation of carbapenemase-producing Enterobacteriaceae (CPE) in many Korean healthcare facilities has rendered the infection-control situation in Korea gloomier, in addition to already prevalent carbapenem-resistant Acinetobacter baumannii (CRAB) [7]. To make matters worse, the recent increase in colistin resistance is extremely worrisome because colistin is the last line of defense against CPE and CRAB [8].

\section{RECENT ANTIMICROBIAL RESISTANCE RATES OF GRAM-NEGATIVE BACILLI}

Accurate nationwide surveillance of antimicrobial-resistant bacteria is becoming more important to establish the optimal treatment guidelines for empirical therapy. The Korean Nosocomial Infections Surveillance System [9], Korean Nationwide Surveillance of Antimicrobial Resistance [10], Korean Network for Study on Infectious Disease, and Korean Antimicrobial Resistance Monitoring System (KARMS) have all played roles in Korea [7]. KARMS was launched by the Korean Center for Disease Control and Prevention (KCDC) in 2002 and reported on the prevalence and characteristics of MDROs from 31 secondary or tertiary hospitals within a finite period. In 2016, the Global Antimicrobial Resistance Surveillance System in Korea was established according to the World Health Organization standards for a global surveillance program to combine patient, laboratory, and epidemiological surveillance data [11].

Table 1 summarizes the recent KARMS data on Enterobacteriaceae [7]. In 2015, the resistance rates of Escherichia coli to ampicillin (72\%), ampicillin-sulbactam $(45 \%)$, cefotaxime (35\%), fluoroquinolone (48\%), gentamycin (29\%), and cotrimoxazole (39\%) were high, while the resistance rates to piperacillin-tazobactam (5\%), cefoxitin (9\%), imipenem (< $0.1 \%)$, and amikacin (1\%) were low. For Klebsiella pneumoniae, the resistance rates to third-generation cephalosporins are similar to those of E. coli, while the resistance rates to piperacillin-tazobactam (21\%) and amikacin (5\%) are higher, and those to gentamicin (19\%) and fluoroquinolone (34\%) are lower, than for E. coli. The resistance rate of K. pneumoniae to tigecycline was $8 \%$. AMRs to major antimicrobials showed no notable change in Enterobacteriaceae between 2013 and 2015.

Table 1 summarizes the recent KARMS data for non-glucose-fermenting GNB [7]. In 2015, the resistance rates of $A$. baumannii imipenem were very high for piperacillin (86\%), piperacillin-tazobactam (82\%), cefotaxime (84\%), cefepime (83\%), imipenem (85\%), amikacin (60\%), gentamycin (75\%), ciprofloxacin (87\%), and cotrimoxazole (64\%). Only tigecycline (4\%) and colistin (< $0.1 \%)$ remain active in A. baumannii. For Pseudomonas aeruginosa, the degree of resistance to piperacillin (27\%), piperacillin-tazobactam (25\%), ceftazidime (19\%), cefepime (18\%), aztreonam (23\%), imipenem (35\%), amikacin (13\%), gentamycin (18\%), and ciprofloxacin (34\%) was less than that for A. baumannii. Colistin activity is also preserved in P. aeruginosa. A. baumannii is one of the main nosocomial pathogens in intensive care units [4], and the dramatic spread of CRAB has resulted in a lack of adequate therapeutic options, except for colistin [12]. Multidrug-resistant features of A. baumannii are a leading threat to immunocompromised hosts and have resulted in high mortality [13].

\section{RESISTANCE MECHANISMS OF ENTEROBAC- TERIACEAE}

According to long-term surveillance by KARMS [7], the resistance of E. coli to ciprofloxacin has increased steadily since 2004. The cefotaxime-resistance rate has increased markedly since 2008, while the cefoxitin-resistance rate has decreased markedly since 2011. For K. pneumoniae, the ciprofloxacin- and cefotaxime-resistance rates have increased since 2004, but not much as in E. coli.

This continuous increase in the antimicrobial resistant rates of E. coli to third- and fourth-generation cephalosporins since the mid-20oos could be due to the spread of the CTX-M type extended-spectrum- $\beta$-lactamase (ESBL)-producing sequence type (ST) 131 clone $[14,15]$. A recent molecular epidemiology study of ST131 E. coli in Korea showed that the ST131 clonal group comprised $21 \%$ of all $268 \mathrm{E}$. coli isolates and $37 \%$ of $57 \mathrm{ST} 131$ isolates had $\mathrm{H}_{3} \mathrm{R} x$ subclones, which showed a significant association with ciprofloxacin and cefotaxime resistance [16]. The spread of ESBL-producing E. coli to 


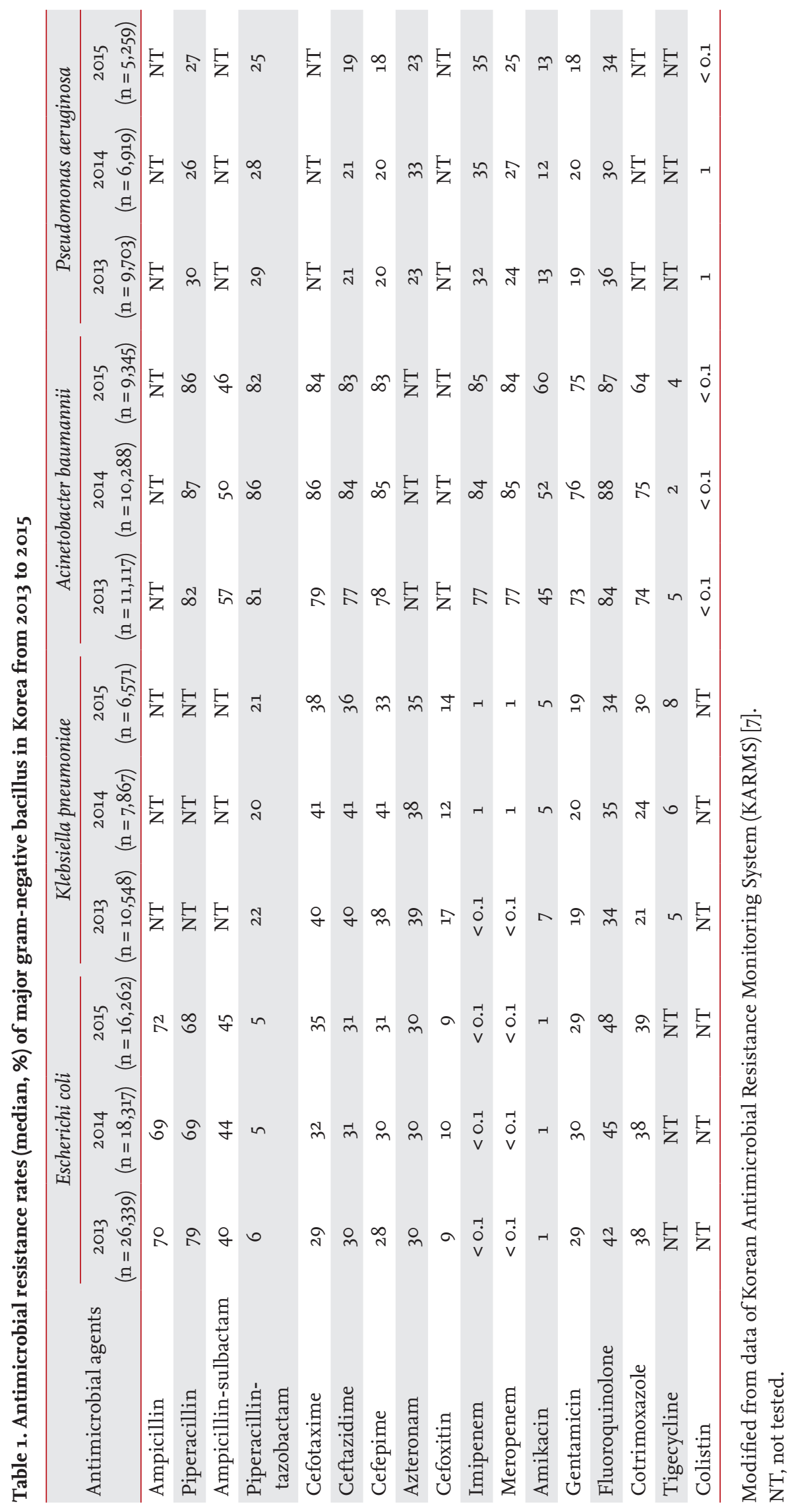


the community is also problematic and the emergence of community-onset bacteremia caused by ESBL-producing E. coli has been a concern in Korea [17]. A study that analyzed 213 E. coli isolates collected from the community found that $25.8 \%$ had CTX-M-type ESBL genes, mainly CTX-M-14 and CTX-M-15, and globally epidemic ST131 clones were found in $27.2 \%$ of the E. coli isolates [18]. With the rapid expansion and dissemination of the ST131 clone in both healthcare facilities and the community, fluoroquinolone resistance has also increased in $E$. coli because the ESBL-producing ST131 clone shares mutations in the fluoroquinolone resistance-determining gyrA and parC regions [19].

In K. pneumoniae, ST11 is the most frequent clone; this is a single-locus variant of the international hyper-epidemic lineage ST258 [20]. The spread of this clone is an emerging threat because the coproduction of carbapenemase (K. pneumoniae carbapenemase 2 [KPC-2]) and $16 \mathrm{~S}$ rRNA methylase ( $r m t B)$, in addition to ESBL, has severely limited antimicrobial therapy [20]. Unlike resistance to aminoglycosides, which is mainly mediated by aminoglycoside modification, 16S rRNA methyltransferase production compromises very potent aminoglycosides with high-level resistance [21]. Resistance to cefoxitin is mediated by AmpC $\beta$-lactamase, resulting from overexpression of the chromosomal AmpC gene or acquisition of a plasmid-mediated AmpC (pAmpC) determinant [22]. Although K. pneumoniae with transferrable pAmpC genes was first detected in Korea in 1988 [23], the cefoxitin-resistance rates of K. pneumoniae have decreased continuously since 2004 [7].

Carbapenem resistance in Enterobacteriaceae (CRE) can result from two different mechanisms. Some CRE that possess either AmpC $\beta$-lactamase or ESBL with concomitant porin mutations can render the organism non-susceptible to carbapenems. More importantly, some CRE may result from the production of carbapenemases that break down carbapenems. Carbapenemases belong to a heterogeneous group of $\beta$-lactamases [24]. The class A penicillinase KPC, class B metalloenzyme New Delhi metallo- $\beta$-lactamase (NDM), and class D oxacillinase (OXA)-type carbapenemases are found in Enterobacteriaceae worldwide [25]. With the global increase in CRE over the past 10 years, the resistance rate to carbapenem in Enterobacteriaceae remains very low in Korea (Table 1). However, the number of CRE isolates increased markedly, from 16 in 2011 to 1,455 in 2016, since KCDC started to monitor CRE in 2010. CRE has also been reported from the community [26]. According to KCDC surveillance data, $K$. pneumoniae is the most common bacterial type of CRE (79.6\% of all isolates in 2015 and $83.2 \%$ in 2016) and carbapenemases were also detected in E. coli, Enterobacter spp., Citrobacter spp., Serratia marcescens, and Klebsiella oxytoca [27]. As shown in Fig. 1, the common Korean genetic types of CRE in 2016 were $\mathrm{KPC}(\mathrm{n}=1,029,70.7 \%), \operatorname{NDM}(\mathrm{n}=197,13.5 \%), \mathrm{OXA}-48$ ( $\mathrm{n}=$ 139, 9.6\%), Guiana-extended spectrum ( $\mathrm{n}=45,3.1 \%)$, Verona integron-encoded metallo- $\beta$-lactamase (VIM; $\mathrm{n}=$ 29, 2.0\%), and imipenemase (IMP; $n=16,1.1 \%$ ) [27]. This situation is mostly due to the increased incidence of CPE due to an outbreak in major healthcare facilities, rendering it a recent public health issue of South Korea [27-29].

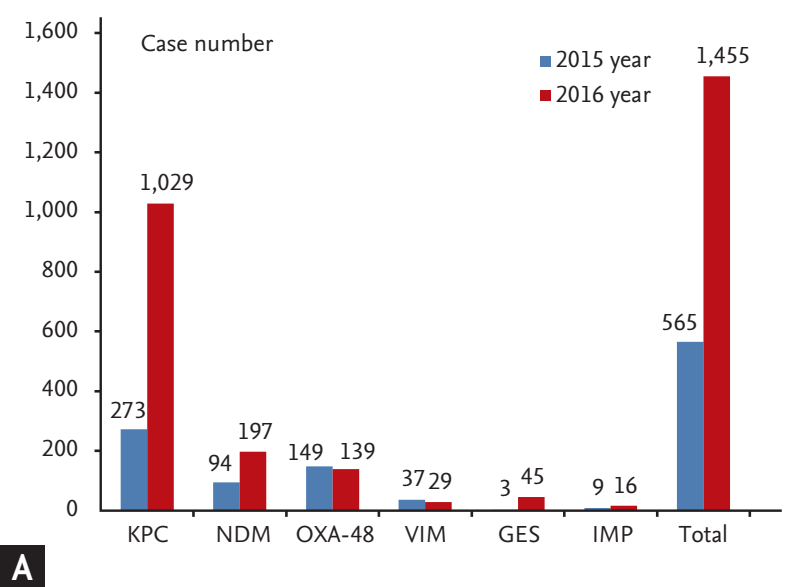

\section{B}

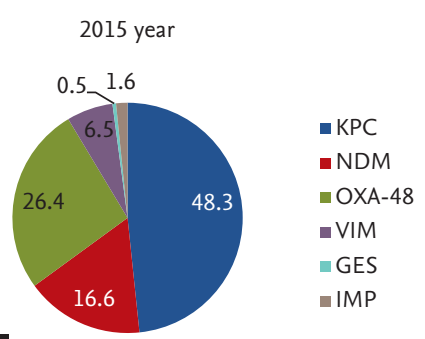

C

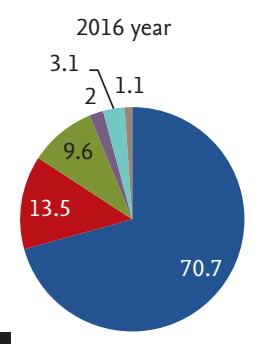

Figure 1. Genetic types of carbapenemase-producing Enterobacteriaceae in South Korea, 2015 to 2016. (A) The reported number of carbapenemase-producing Enterobacteriaceae in Korea. (B, C) Genotype distribution of carbapenemase-producing Enterobacteriaceae in Korea. KPC, Klebsiella pneumoniae carbapenemase; NDM, New Delhi metallo- $\beta$-lactamase; OXA-48, oxacillinase-48; VIM, Verona integron-encoded metallo- $\beta$-lactamase; GES, Guiana-extended spectrum; IMP, imipenemase. 


\section{RESISTANCE MECHANISMS OF NON-GLU- COSE-FERMENTING GRAM-NEGATIVE BACILLI}

The resistance rate of $A$. baumannii to imipenem increased to $85 \%$ in 2015 [7], representing a major public threat in Korea. Carbapenem is usually considered a treatment option for ESBL producers and the increased nationwide rate of prescription of carbapenems is correlated with the rapid rise in CRAB isolation since 2007 [30]. OXA-type $\beta$-lactamases are the main resistance mechanism for CRAB and a drastic increase in Acinetobacter isolates with $b l a_{\text {OXA-23 }}$ has been observed since the mid-200os, whereas ISAba1-associated $b l a_{\text {OXA-51 }}$, another contributor to CRAB, has decreased since the mid-200os [31]. Many CRAB isolates are multidrug-resistant or extensively drug-resistant because they usually co-carry AMR through armA/aminoglycosides-modifying enzymes (aminoglycosides), DNA gyrase/topoisomerase IV (fluoroquinolones), and Ade-type efflux pumps (tetracyclines) [32]. Recently, the treatment of carbapenem-resistant A. baumannii infection has been severely restricted because the agents of last resort, such as colistin and tigecycline, are losing their efficacy with the emergence of colistin resistance in A. baumannii. The colistin-resistant gene of Acinetobacter spp. is usually located in the chromosome and the mechanisms of resistance are modification of lipid A by phosphoethanolamine via $\mathrm{pmr} A \mathrm{~B}$ two-component regulatory gene mutations (pmrA and pmrB) and loss of lipopolysaccharide via inactivation of a lipid A biosynthesis gene (lpxA, lpxC, or lpxD) [8].

The resistance rate of $P$. aeruginosa to imipenem was $35 \%$ in 2015 , but no marked rise in resistance was observed in P. aeruginosa from 2004 to 2015 [7]. Since the first report of VIM-2-producing P. aeruginosa in Korea in 2002 [33], metallo- $\beta$-lactamases have been the main mechanism for carbapenem-resistant $P$. aeruginosa in the IMP and VIM types [34]. The rates of amikacin- and gentamicin-resistant $P$. aeruginosa have declined continuously [7] and this is correlated with the Korea-wide reduction in aminoglycoside use [35]. Aminoglycosides, except amikacin, are considered old drugs, but they still play key roles in the treatment of infections, and have potent bactericidal activity against some CRE [36].

\section{TREATMENT OPTIONS FOR ANTIMICROBI- AL-RESISTANT GRAM-NEGATIVE BACTERIA}

There are relatively few treatment options for CRAB and CRE. Most reports are retrospective observational studies. Because there are little published data from randomized controlled trials, more data on the antimicrobial treatment of CRAB or CRE are needed to make treatment recommendations. The mortality associated with invasive CRAB infection is high and is linked to comorbid disease severity and initial inappropriate antimicrobial therapy [37]. Moreover, infection with, or colonization of, antimicrobial-resistant Gram-negative bacteria is associated with a longer hospital stay and increased medical costs $[38,39]$. Although extensively drug-resistant (resistant to all but two or less classes) isolates have been reported, the majority of isolates remain susceptible to polymyxin (colistin or polymyxin B). Many retrospective studies and meta-analyses have shown that combination therapy confers a survival advantage over monotherapy [40-43].

\section{POLYMYXINS}

Polymyxins interact with lipid A in the Gram-negative bacterial outer membrane, leading to the leakage of cellular contents and bacterial cell death. With increasing antibiotic resistance among important Gram-negative bacteria and the lack of new antibiotics, old antibiotics discovered in the 1950 s have been reintroduced in recent years [44]. Polymyxin B and colistin (polymyxin E) are two polymyxins that can be used. Colistin is administered as the inactive prodrug colistin methanesulfonate (CMS), whereas polymyxin $\mathrm{B}$ is administered as an active drug. CMS requires a loading dose of up to 300 $\mathrm{mg}$ (colistin base activity) because it has to be converted into colistin in the plasma and patients are otherwise exposed to suboptimal concentrations for several days. Although polymyxin B is not available in Korea, it has superior pharmacological characteristics for treating infections and rapidly and reliably achieves the desired plasma concentration of the active polymyxin [45,46]. Combination therapy with a second active agent or an agent that demonstrates synergy with colistin is recommended. The two major complications associated with 
polymyxins are nephrotoxicity and neurotoxicity. Approximately $50 \%$ of cases manifest variable degrees of nephrotoxicity due to colistin, although these are usually reversible.

\section{CARBAPENEMS}

Carbapenems are considered the drug of choice for treating infections caused by multidrug-resistant Gram-negative bacteria due to their excellent activity against these organisms and safety profile. However, there is concern about carbapenem use because of the increasing resistance to them. High-dose meropenem (6,000 mg/day) administered by prolonged ( $>4$ hours) infusion had a high probability of target attainment in Monte Carlo dosing simulations [47]. Although the pharmacokinetic data appear favorable [48], therapy with carbapenem alone cannot be recommended because there are limited clinical data. Carbapenem-based combination therapy resulted in relatively low mortality in retrospective studies $[42,49]$. High-dose, extended infusion of a carbapenem as part of a combination regimen can be considered a viable treatment for carbapenem-resistant organisms.

\section{TIGECYCLINE}

Tigecycline is a glycylcycline antimicrobial and a semisynthetic derivative of minocycline. This newly developed antimicrobial inhibits protein synthesis and is bacteriostatic. Tigecycline is approved for the treatment of skin and skin structure infections, complicated intra-abdominal infections, and community-acquired pneumonia. Outcomes with tigecycline monotherapy have not been favorable. High dosing of tigecycline, i.e., increasing the dose of tigecycline to $100 \mathrm{mg}$ twice daily, should be considered because of the low likelihood of reaching target pharmacokinetic/pharmacodynamic parameters with the conventional dose of $50 \mathrm{mg}$ twice daily. Tigecycline should be reserved for cases with no other treatment options due to its black-box warning of increased mortality [42].

\section{COMBINATION THERAPY}

Monotherapy for CRE is not better than inappropriate therapy, and combination therapy offers a survival advantage over monotherapy [42]. Moreover, monotherapy can lead to resistance and possible loss of that class of antimicrobials. The combinations associated with the lowest mortality rates contained a carbapenem [42]. Several retrospective studies have documented lower mortality rates after CRAB infection when more than one agent was given for therapy $[40,41]$. However, it is still controversial whether combined therapy is more effective than monotherapy [50].

\section{CONCLUSIONS}

AMR is increasing among Gram-negative bacteria in Korea. Treatment options for drug-resistant Gram-negative bacteria, such as CRE and CRAB, are limited. We are on the cusp of a post-antibiotics era. To optimize treatment, continued research on combination therapy and the dosing of antimicrobials is needed. New drug development, and prolongation of the activity of existing antimicrobials through appropriate use, have become important strategies.

\section{Conflict of interest}

No potential conflict of interest relevant to this article was reported.

\section{REFERENCES}

1. Martin-Loeches I, Diaz E, Valles J. Risks for multidrug-resistant pathogens in the ICU. Curr Opin Crit Care 2014;20:516-524.

2. Yoon YK, Park GC, An H, Chun BC, Sohn JW, Kim MJ. Trends of antibiotic consumption in Korea according to national reimbursement data (2008-2012): a population-based epidemiologic study. Medicine (Baltimore) 2015;94:e2100.

3. Kim DS, Jang SM, Kim NS. Epidemiologic investigation on antibiotic use using defined daily dose. J Korean Acad Manag Care Pharm 2010;2:47-59.

4. Lee Y, Kim YA, Song W, et al. Recent trends in antimicro- 
bial resistance in intensive care units in Korea. Korean J Nosocomial Infect Control 2014;19:29-36.

5. Abbasi J. Infectious disease expert sees threat from colistin-resistant superbug. JAMA 2016;316:806-807.

6. Ryu S. The new Korean action plan for containment of antimicrobial resistance. J Glob Antimicrob Resist 2017;8:70-73.

7. Kim D, Ahn JY, Lee CH, et al. Increasing resistance to extended-spectrum cephalosporins, fluoroquinolone, and carbapenem in gram-negative bacilli and the emergence of carbapenem non-susceptibility in Klebsiella pneumoniae: analysis of Korean Antimicrobial Resistance Monitoring System (KARMS) data from 2013 to 2015. Ann Lab Med 2017;37:231-239.

8. Cai Y, Chai D, Wang R, Liang B, Bai N. Colistin resistance of Acinetobacter baumannii: clinical reports, mechanisms and antimicrobial strategies. J Antimicrob Chemother 2012;67:1607-1615.

9. Kim EJ, Kwak YG, Park SH, et al. Trends of device utilization ratios in intensive care units over 10-year period in South Korea: device utilization ratio as a new aspect of surveillance. J Hosp Infect 2017.

10. Yong D, Shin HB, Kim YK, et al. Increase in the prevalence of carbapenem-resistant Acinetobacter isolates and ampicillin-resistant non-typhoidal salmonella species in Korea: a KONSAR Study conducted in 2011. Infect Chemother 2014;46:84-93.

11. World Health Organization. Global antimicrobial resistance surveillance system [Internet]. Geneva: World Health Organization, c2018 [cited 2018 Feb 8]. Available from: http://www.who.int/antimicrobial-resistance/ publications/surveillance-system-manual/en/.

12. Jasemi S, Douraghi M, Adibhesami H, et al. Trend of extensively drug-resistant Acinetobacter baumannii and the remaining therapeutic options: a multicenter study in Tehran, Iran over a 3-year period. Lett Appl Microbiol 2016;63:466-472.

13. Moon SY, Peck KR, Chang HH, et al. Clinical experience of tigecycline treatment in infections caused by extensively drug-resistant Acinetobacter spp. Microb Drug Resist 2012;18:562-566.

14. Johnson JR, Johnston B, Clabots C, Kuskowski MA, Castanheira M. Escherichia coli sequence type ST131 as the major cause of serious multidrug-resistant E. coli infections in the United States. Clin Infect Dis 2010;51:286-
294.

15. Rogers BA, Sidjabat HE, Paterson DL. Escherichia coli O25b-ST131: a pandemic, multiresistant, community-associated strain. J Antimicrob Chemother 2011;66:1-14.

16. Kim SY, Park YJ, Johnson JR, Yu JK, Kim YK, Kim YS. Prevalence and characteristics of Escherichia coli sequence type 131 and its $\mathrm{H}_{3} \mathrm{O}$ and $\mathrm{H}_{3} \mathrm{ORx}$ subclones: a multicenter study from Korea. Diagn Microbiol Infect Dis 2016;84:97-101.

17. Kim H, Kim YA, Park YS, Choi MH, Lee GI, Lee K. Risk factors and molecular features of sequence type (ST) 131 extended-spectrum $\beta$-lactamase-producing Escherichia coli in community-onset bacteremia. Sci Rep 2017;7:14640.

18. Kim YA, Kim JJ, Kim H, Lee K. Community-onset extended-spectrum- $\beta$-lactamase-producing Escherichia coli sequence type 131 at two Korean community hospitals: the spread of multidrug-resistant E. coli to the community via healthcare facilities. Int J Infect Dis 2017;54:39-42.

19. Johnson JR, Tchesnokova V, Johnston B, et al. Abrupt emergence of a single dominant multidrug-resistant strain of Escherichia coli. J Infect Dis 2013;207:919-928.

20. DSouza R, Pinto NA, Hwang I, et al. Molecular epidemiology and resistome analysis of multidrug-resistant ST11 Klebsiella pneumoniae strain containing multiple copies of extended-spectrum $\beta$-lactamase genes using whole-genome sequencing. New Microbiol 2017;40:38-44.

21. Wachino J, Arakawa Y. Exogenously acquired 16S rRNA methyltransferases found in aminoglycoside-resistant pathogenic Gram-negative bacteria: an update. Drug Resist Updat 2012;15:133-148.

22. Pfeifer Y, Cullik A, Witte W. Resistance to cephalosporins and carbapenems in Gram-negative bacterial pathogens. Int J Med Microbiol 2010;300:371-379.

23. Bauernfeind A, Chong Y, Schweighart S. Extended broad spectrum beta-lactamase in Klebsiella pneumoniae including resistance to cephamycins. Infection 1989;17:316321.

24. Queenan AM, Bush K. Carbapenemases: the versatile beta-lactamases. Clin Microbiol Rev 2007;20:440-458.

25. Bonomo RA, Burd EM, Conly J, et al. Carbapenemase-producing organisms: a global scourge! Clin Infect Dis 2017 .

26. Lee HJ, Choi JK, Cho SY, et al. Carbapenem-resistant enterobacteriaceae: prevalence and risk factors in a single community-based hospital in Korea. Infect Chemother 
2016;48:166-173.

27. Kim MN, Yong D, An D, et al. Nosocomial clustering of NDM-1-producing Klebsiella pneumoniae sequence type 340 strains in four patients at a South Korean tertiary care hospital. J Clin Microbiol 2012;50:1433-1436.

28. Jeong SH, Lee KM, Lee J, et al. Clonal and horizontal spread of the blaOXA-232 gene among Enterobacteriaceae in a Korean hospital. Diagn Microbiol Infect Dis 2015;82:70-72.

29. Hong SK, Yong D, Kim K, et al. First outbreak of KPC-2-producing Klebsiella pneumoniae sequence type 258 in a hospital in South Korea. J Clin Microbiol 2013;51:38773879 .

30. Kim YA, Park YS, Youk T, Lee H, Lee K. Abrupt increase in rate of imipenem resistance in Acinetobacter baumannii complex strains isolated from general hospitals in Korea and correlation with carbapenem administration during 2002-2013. Ann Lab Med 2018;38:179-181.

31. Lee Y, Kim YR, Kim J, et al. Increasing prevalence of blaOXA-23-carrying Acinetobacter baumannii and the emergence of blaOXA-182-carrying Acinetobacter nosocomialis in Korea. Diagn Microbiol Infect Dis 2013;77:160-163.

32. Doi Y, Murray GL, Peleg AY. Acinetobacter baumannii: evolution of antimicrobial resistance-treatment options. Semin Respir Crit Care Med 2015;36:85-98.

33. Lee K, Lim JB, Yum JH, et al. bla(VIM-2) cassette-containing novel integrons in metallo-beta-lactamase-producing Pseudomonas aeruginosa and Pseudomonas putida isolates disseminated in a Korean hospital. Antimicrob Agents Chemother 2002;46:1053-1058.

34. Hong DJ, Bae IK, Jang IH, Jeong SH, Kang HK, Lee K. Epidemiology and characteristics of metallo- $\beta$-lactamase-producing Pseudomonas aeruginosa. Infect Chemother 2015;47:81-97.

35. Kim YA, Park YS, Youk T, Lee H, Lee K. Correlation of aminoglycoside consumption and amikacin- or gentamicin-resistant Pseudomonas aeruginosa in long-term nationwide analysis: is antibiotic cycling an effective policy for reducing antimicrobial resistance? Ann Lab Med 2018;38:176-178.

36. Almaghrabi R, Clancy CJ, Doi Y, et al. Carbapenem-resistant Klebsiella pneumoniae strains exhibit diversity in aminoglycoside-modifying enzymes, which exert differing effects on plazomicin and other agents. Antimicrob Agents Chemother 2014;58:4443-4451.

37. Lemos EV, de la Hoz FP, Einarson TR, et al. Carbapenem resistance and mortality in patients with Acinetobacter baumannii infection: systematic review and meta-analysis. Clin Microbiol Infect 2014;20:416-423.

38. Lee NY, Lee HC, Ko NY, et al. Clinical and economic impact of multidrug resistance in nosocomial Acinetobacter baumannii bacteremia. Infect Control Hosp Epidemiol 2007;28:713-719.

39. Lee H, Lee H. Clinical and economic evaluation of multidrug-resistant Acinetobacter baumannii colonization in the intensive care unit. Infect Chemother 2016;48:174-180.

40. Batirel A, Balkan II, Karabay O, et al. Comparison of colistin-carbapenem, colistin-sulbactam, and colistin plus other antibacterial agents for the treatment of extremely drug-resistant Acinetobacter baumannii bloodstream infections. Eur J Clin Microbiol Infect Dis 2014;33:1311-1322.

41. Shields RK, Clancy CJ, Gillis LM, et al. Epidemiology, clinical characteristics and outcomes of extensively drug-resistant Acinetobacter baumannii infections among solid organ transplant recipients. PLoS One 2012;7:e52349.

42. Tzouvelekis LS, Markogiannakis A, Piperaki E, Souli M, Daikos GL. Treating infections caused by carbapenemase-producing Enterobacteriaceae. Clin Microbiol Infect 2014;20:862-872.

43. Falagas ME, Lourida P, Poulikakos P, Rafailidis PI, Tansarli GS. Antibiotic treatment of infections due to carbapenem-resistant Enterobacteriaceae: systematic evaluation of the available evidence. Antimicrob Agents Chemother 2014;58:654-663.

44. Falagas ME, Kasiakou SK. Colistin: the revival of polymyxins for the management of multidrug-resistant gram-negative bacterial infections. Clin Infect Dis 2005;40:1333-1341.

45. Nation RL, Velkov T, Li J. Colistin and polymyxin B: peas in a pod, or chalk and cheese? Clin Infect Dis 2014;59:8894.

46. Kassamali Z, Danziger L. To B or not to B, that is the question: is it time to replace colistin with polymyxin B? Pharmacotherapy 2015;35:17-21.

47. Roberts JA, Kirkpatrick CM, Roberts MS, Robertson TA, Dalley AJ, Lipman J. Meropenem dosing in critically ill patients with sepsis and without renal dysfunction: intermittent bolus versus continuous administration? Monte Carlo dosing simulations and subcutaneous tissue distribution. J Antimicrob Chemother 2009;64:142-150.

48. MacVane SH, Kuti JL, Nicolau DP. Prolonging $\beta$-lactam infusion: a review of the rationale and evidence, and 
guidance for implementation. Int J Antimicrob Agents 2014;43:105-113.

49. Qureshi ZA, Paterson DL, Potoski BA, et al. Treatment outcome of bacteremia due to KPC-producing Klebsiella pneumoniae: superiority of combination antimicrobial regimens. Antimicrob Agents Chemother 2012;56:2108-
2113.

50. Lopez-Cortes LE, Cisneros JM, Fernandez-Cuenca F, et al. Monotherapy versus combination therapy for sepsis due to multidrug-resistant Acinetobacter baumannii: analysis of a multicentre prospective cohort. J Antimicrob Chemother 2014;69:3119-3126. 